\section{BMJ Open Respiratory Research}

\title{
Post-tuberculous fibrosing mediastinitis: a review of the literature
}

\author{
Zhe Wu, ${ }^{1}$ Hannah Jarvis, ${ }^{2}$ Luke S Howard, ${ }^{3}$ Corrina Wright, ${ }^{4}$ Onn Min Kon ${ }^{1}$
}

To cite: Wu Z, Jarvis $\mathrm{H}$, Howard LS, et al. Posttuberculous fibrosing mediastinitis: a review of the literature. BMJ Open Resp Res 2017;4:e000174. doi:10.1136/ bmjresp-2016-000174

Received 1 December 2016 Revised 8 March 2017 Accepted 13 March 2017

\section{CrossMark}

${ }^{1}$ Department of Respiratory Medicine, St Mary's Hospital, Imperial College Healthcare NHS Trust, London, UK ${ }^{2}$ Imperial Clinical Respiratory Research Unit, Imperial College London, London, UK ${ }^{3}$ National Pulmonary Hypertension Service, Imperial College Healthcare NHS Trust, London, UK ${ }^{4}$ Department of Cytopathology, St Mary's Hospital, Imperial College Healthcare NHS Trust, London, UK

Correspondence to Dr Onn Min Kon; onn.kon@nhs.net

\section{ABSTRACT}

Fibrosing mediastinitis (FM) is a rare disease where there is thickening of the fibrous tissue in the mediastinum. While histoplasmosis is the the most common recognised cause, the link with tuberculosis (TB) has been rarely documented. We review the link between TB and FM, and describe a case of probable TB-related FM.Our case is of a 74-year-old man who presented with breathlessness 3 years after fully treated TB. Scans revealed a calcified soft tissue mass within the mediastinum, and a diagnosis of fibrosing mediastinitis resulting in pulmonary hypertension was made. Tests for histoplasmosis and IgG4 staining were negative. Surgical intervention was not felt to be beneficial, and he was treated with prednisolone and mycophenolate mofetil.In the review, we highlight the two forms of mediastinitis-granulomatous versus fibrous, and how these two entities may be on a spectrum of disease progression. We also explore the prevalence, clinical presentation, pathogenesis, imaging techniques and treatment options of TBrelated FM.

\section{INTRODUCTION}

Fibrosing mediastinitis (FM) or mediastinal fibrosis is a rare disease where thickening of the fibrous tissue in the mediastinum leads to an array of complications ranging from a benign clinical course to obstruction of vascular, airway and oesophageal structures. ${ }^{1}$

The most common cause linked to this condition is a secondary reaction to histoplasmosis, with reports mainly in the USA. However, even among patients with histoplasmosis infection, only 3 in 100000 were found to develop fibrosing mediastinitis. ${ }^{2}$ Other conditions linked to FM include tuberculosis (TB) ${ }^{3}$ aspergillosis ${ }^{45}$ and more recently IgG4 related disease. ${ }^{6}$ An idiopathic form, with non-calcific fibrosis, has also been described and can be associated with extrathoracic fibrotic conditions such as retroperitoneal fibrosis as well as other autoimmune disease. ${ }^{78}$

The literature describes two forms of mediastinitis, namely granulomatous mediastinitis, where fibrous reaction is limited to the mediastinal lymph nodes, and fibrosing mediastinitis, where there is a more widespread reaction in the mediastinal space. ${ }^{3}$ The distinction between these two forms of disease is explored in this article.

Despite the link between TB and FM, such cases are sparsely reported in the literature. In this paper, we present a new case of TB-related mediastinal fibrosis and provide a literature review of the association between these two conditions.

\section{CASE PRESENTATION}

A 74-year-old man, of Asian origin, with a medical history including a myocardial infarction in 2007 and fully sensitive treated tuberculosis in 2010, presented in March 2013 in Pakistan with a 4-week history of breathlessness.

His examination findings of the chest revealed mild expiratory wheeze and reduced air entry in the left base. A CT scan showed apical scarring bilaterally, with multiple small nodules in the right middle and lower lobes, the largest measuring $4 \mathrm{~mm}$ and a left-sided pleural effusion. Pleural aspiration removed $500 \mathrm{~mL}$ of transudative fluid, and cultures were negative for TB. Sputum culture was also negative for TB. He was treated with a week's course of antibiotics, steroids and inhalers with a diagnosis of asthma. After dischargehe was weaned off the steroids and inhalers as he did not feel any subjective benefit.

In October 2013, he was admitted again into hospital following a recurrence of the left pleural effusion, this time in the UK. Lung function testing showed a mixed obstructive and restrictive pattern with forced expiratory volume in $1 \mathrm{~s} 1.1 \mathrm{~L}$ (50\% predicted), forced vital capacity $1.8 \mathrm{~L}(60 \%)$ and transfer factor for carbon monoxide 3.69 (50\%). Echocardiography showed an estimated left ventricular ejection fraction of $60 \%$. However, he had developed a mildly dilated right atrium and right ventricle with mild right ventricular hypertrophy, and moderately elevated pulmonary artery systolic pressure of $60 \mathrm{~mm} \mathrm{Hg}$. Coronary angiogram showed patent stents. He was, therefore, referred to the regional centre for pulmonary hypertension and 


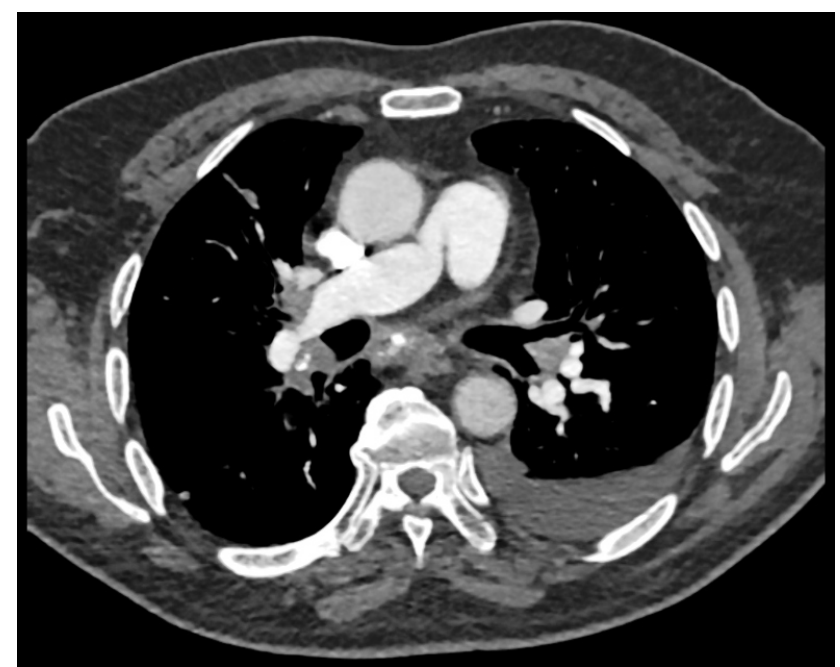

Figure 1 CT scan showing a soft tissue mass with multiple areas of calcification, causing narrowing of the right pulmonary artery. A left-sided small pleural effusion is also present.

subsequently underwent right heart catheterisation which showed a mean pulmonary artery pressure of $31 \mathrm{~mm} \mathrm{Hg}$, pulmonary wedge pressure of $10 \mathrm{~mm} \mathrm{Hg}$ and pulmonary vascular resistance of 512 dynes $\cdot \mathrm{sec} \cdot \mathrm{cm}^{-5}$ in keeping with precapillary pulmonary hypertension.

Crucially, on repeat CT (see figure 1), there was a new calcified soft tissue mass within the mediastinum and hilar region, and a diagnosis of FM was made.

An expert opinion was sought regarding surgical intervention, and it was felt that the mediastinal fibrosis was causing extrinsic vascular compression with multifocal proximal pulmonary artery stenoses and narrowing of the pulmonary veins at the bronchial level. However, angioplasty was considered too high risk for arterial rupture as multiple vessels were affected.

A fluorodeoxyglucose-positron emission tomography (FDG-PET) scan (see figure 2A,B) revealed a metabolically active partially calcified hilar and mediastinal soft tissue mass with activity. The patient underwent an endobronchial ultrasound in May 2014 and histology from the lymph nodes (see figure $3 \mathrm{~A}, \mathrm{~B}$ ) revealed necrotic debris, anthracotic macrophages and occasional lymphoid cells without evidence of granuloma or malignant cells and negative IgG4 staining. TB culture and PCR were both negative, as were tests for histoplasma, antinuclear antibody and HIV. Due to the increased activity seen on PET scan, he was started on $60 \mathrm{mg}$ prednisolone daily in June 2014 with TB chemoprophylaxis.

After 2 months of treatment, he had symptomatically improved, back almost to his baseline in 2007. However, a repeat CT scan did not show any significant difference to the previous scan.

After reducing the prednisolone to $50 \mathrm{mg}$, his breathing declined and was admitted again in September 2014 for progressive breathlessness. He was given three doses of intravenous methylprednisolone, another therapeutic aspiration of $500 \mathrm{~mL}$ of the left-sided effusion and discharged on prednisolone $40 \mathrm{mg}$ and furosemide. To wean the steroid dose, he was started on mycophenolate mofetil (MMF) $500 \mathrm{mg}$ twice daily in October 2014. By November 2014, he was taking only $10 \mathrm{mg}$ prednisolone daily. During the subsequent months, he remained steroid dependent but had complications with steroid-related side effects including osteoporotic vertebral fractures and myopathy.
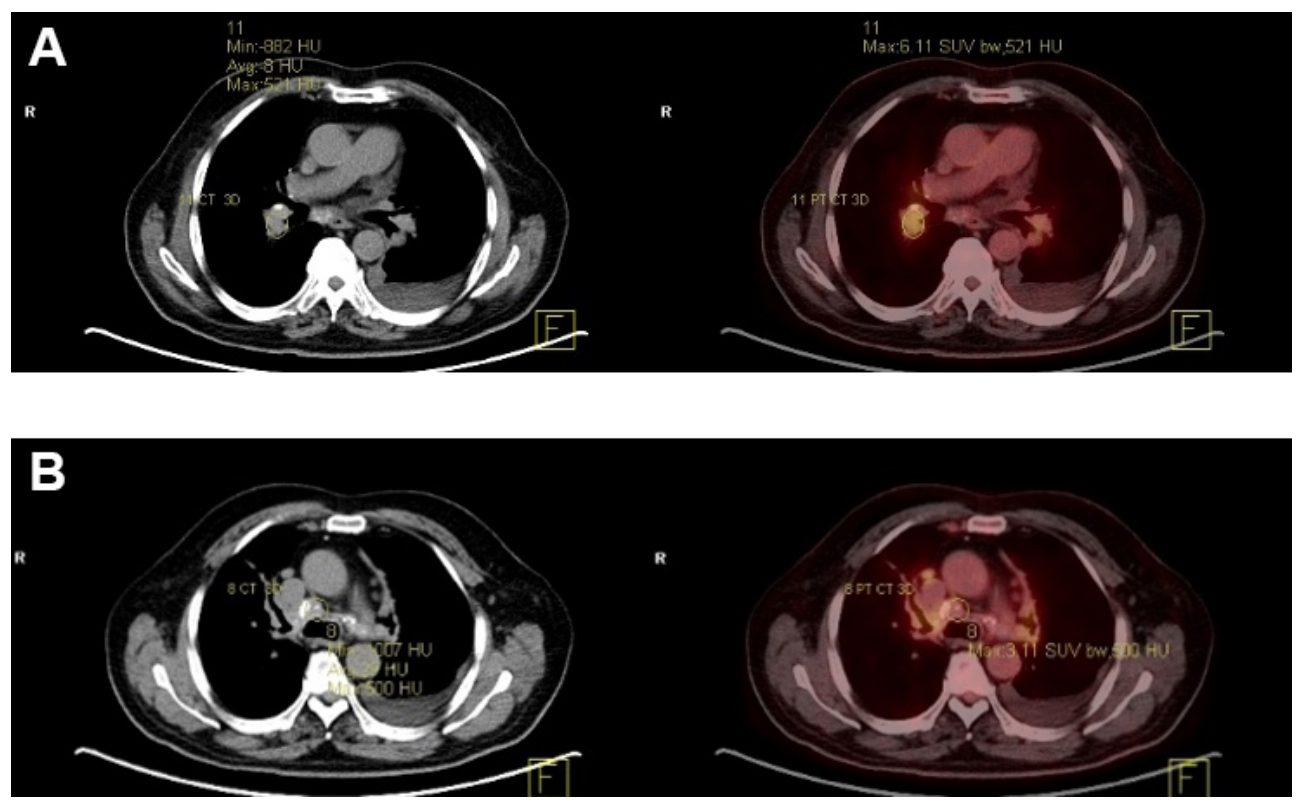

Figure 2 CT (left of images) showing calcified areas and PET (right of images) scans showing increased FDG-PET metabolic activity in the mediastinal mass (A) and perihilar lymph nodes (B). FDG-PET, fluorodeoxyglucose-positron emission tomography. 

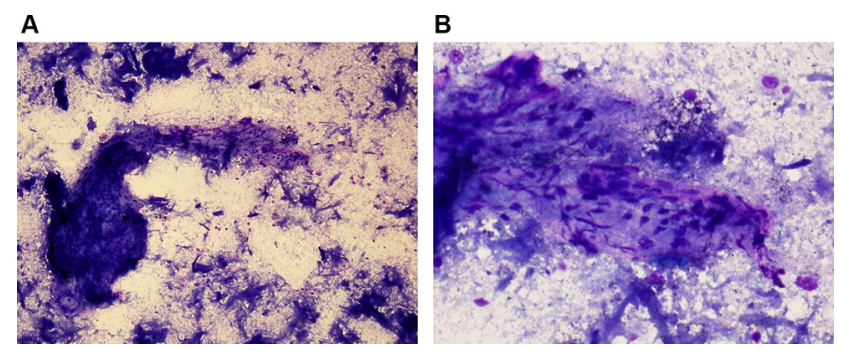

Figure 3 Microbiopsy of mediastinal mass showing fibrous tissue containing spindle cells, with anthracotic macrophages and necrotic debris in the background, May Graunwald Giemsa (MGG) stain. MGG x100 (A), MGG ×400 (B).

In January 2015, he developed marked lethargy with peripheral oedema and was admitted again for intravenous methylprednisolone, diuresis and a further 660 $\mathrm{mL}$ of transudative fluid was aspirated from the left-sided effusion. A repeat echocardiogram showed worsening pulmonary hypertension with a pulmonary artery systolic pressure of $78 \mathrm{~mm} \mathrm{Hg}$. Nevertheless, his symptoms have remained stable on $5 \mathrm{mg}$ prednisolone and $500 \mathrm{mg}$ twice daily MMF, with a stable effusion and his exercise tolerance is between 300 and $400 \mathrm{~m}$.

\section{Prevalence}

The prevalence of TB-related mediastinal fibrosis is unknown, with only a handful of cases having been reported in the literature. In a large case review of 180 cases by Schowengerdt et al in 1969, there were 13 cases of granulomatous mediastinitis and only one case of FM where TB was identified as the culprit (via confirmation of acid-fast bacilli). The authors reviewed cases that, at the time, were not subjected to current testing methods for TB infection such as TB-PCR. However, the discrepancy between the rates of TB found in the granulomatous versus fibrosing disease spectrum may support the concept of the two states existing in a dynamic spectrum with granulomatous mediastinitis being seen in early active infection, where AFBs are more likely to be detected. Only a proportion of granulomatous mediastinitis cases lead to FM, where the scarring invades into the rest of the mediastinum. Therefore, FM could represent the later stages of an aberrant healing response following an active infection. This theory is observed in many subsequent case reports where tissue from fibrotic mediastinum has yielded negative results for AFB and TB cultures. Indeed, this is also observed in Histoplasmosis-related fibrosis, where most cases are also culture negative. ${ }^{9}$ Nevertheless, it is important to highlight that due to the rarity of the disease, it is not clear from the literature whether the two forms of mediastinitis represent ends of a spectrum or distinct clinical entities.

A literature search for other case reports documented in English revealed five single case reports ${ }^{10-14}$ and two case series. The first case series, by Mole and colleagues, ${ }^{15}$ detailed eight patients with TB-related FM and a further single case with both TB and histoplasmosis. The second case series reported a further nine patients with active pulmonary TB and dysphagia attributed to FM. ${ }^{16}$ The basis for the diagnosis of FM in all of these cases was plain chest radiography alongside normal gastroscopy. A more definitive diagnosis using either CT imaging and/ or mediastinal tissue biopsy was not obtained.

\section{Clinical presentation}

The initial presentation of FM depends on the anatomical structures that are affected by the fibrotic reaction. Schowengerdt et $a \hat{l}$ identified that in early granulomatous disease, only a quarter $(26 \%)$ of patients had involvement of adjacent structures, compared with the later fibrotic stage where this percentage increased over threefold $(83 \%)$. The most common structure involved was the superior vena cava (SVC), with over $60 \%$ of the patients with FM having caval obstruction. ${ }^{3}$ Other regions involved included the oesophagus, trachea, bronchi, pulmonary vein and artery, inferior vena cava, thoracic duct, atrium and recurrent laryngeal nerve. ${ }^{3}$ SVC obstruction was also reported by the Mayo Clinic ( 6 out of 31 cases), ${ }^{17}$ and by Mole and colleagues (6 out of 18). ${ }^{15}$

Although SVC obstruction is a feature, the slowly progressive nature of this condition allows collateral vein formation, and patients may often be asymptomatic initially. Progression of disease may lead to shortness of breath, venous distension of the neck and upper chest region, facial oedema and features of cor pulmonale. Should the bronchial structures be involved, there may be progressive dyspnoea, cough and haemoptysis. Other complaints include dysphagia with oesophageal invasion, hoarseness of voice with recurrent laryngeal nerve entrapment, as well as non-specific chest pain and malaise. ${ }^{15}$

Of the single case reports where FM is specifically believed to be related to prior TB infection, presentations were due to superior vena cava syndrome, ${ }^{10} 11$ bronchial obstruction, ${ }^{12}$ pulmonary artery compression, ${ }^{13}$ as well as impingement of the transverse sinus and its adjacent vascular structures. $^{14}$

\section{Pathogenesis}

The pathogenesis of fibrotic tissue formation in FM is not clear. It has been proposed with Histoplasmosis infection, that the organism spreads along the lymphatic system and a cell-mediated response drives the formation of mediastinal granulomas.

Flieder and colleagues examined the histological morphology of tissue taken from 30 cases of idiopathic FM and proposed the condition as a three-stage progression of fibroinflammatory changes. Stage I was characterised by oedematous fibromyxoid tissue with numerous spindle cells, eosinophils, mast cells, lymphocytes and plasma cells. Stage II developed into thick bands of haphazardly arranged collagen. Stage III revealed dense acellular collagen and occasional dystrophic calcification. ${ }^{18}$ Interestingly, a more recent study has revealed 
that CD20-positive B lymphocytes are commonly seen on immunophenotyping and may be involved in potentiating disease progression. ${ }^{19}$

\section{Diagnosis}

Several methods have been reported for obtaining the diagnosis of FM. Prior to the use of more advanced imaging techniques, a chest radiograph was a useful way of raising suspicion, with widening of the mediastinum and lymphadenopathy of the hilar and paratracheal regions. ${ }^{20}$ The mainstay of imaging currently is CT. The majority of cases with FM will have localised fibrotic areas with calcification and there is much better identification of the surrounding vascular and airway compression with this imaging modality ${ }^{20}{ }^{21}$ In some cases, a diffuse soft tissue mass without calcification can also be seen, ${ }^{22}$ and tissue sampling for malignancy should be sought. Although mediastinoscopy and surgical exploration are methods to obtain tissue, ${ }^{3} 15$ we used the less invasive technique of endobronchial ultrasound.

MRI has equal effect in recognising mediastinal structural involvement, but importantly, it is not as specific for identifying calcification, which is the hallmark of FM. ${ }^{23}$ MRI obviates the need for intravenous contrast for prominent vascular structures, which may be advantageous in those patients who are iodine allergic or who have severe renal impairment.

There have been some reports of using pulmonary scintigraphy ${ }^{24-26}$ to visualise perfusion defects and angiography to identify vascular compromise, but they do not diagnose FM, rather they are adjuncts to CT or MRI. In addition, CT or MRI angiography can in the modern era demonstrate sufficient detail of vessel encroachment without such tests. ${ }^{14}$

The role of FDG PET/CT scanning has been unclear. Chong et al demonstrated negative FDG uptake, concluding that PET scanning is useful in differentiating with malignancy. ${ }^{27}$ However, others have shown that active inflammatory lesions are also PET avid, so it can used to gauge response to treatment, although biopsy to exclude a neoplastic process is still mandatory. ${ }^{28} 29$

\section{Treatment and clinical outcomes}

The management of FM is divided into surgical and medical therapy. The surgical approach depends on the anatomical structure involved, but is used as a last resort as fibrosis surrounding vital structures makes the procedure extremely challenging.

In the few reported cases of TB-related FM highlighted in the beginning of this review, outcomes have been variable. In the case reported by Ojeifo et $a l$, the patient was treated for latent TB for 6 months on the basis of a positive interferon- $\gamma$ release assay with no evidence of active disease from microbiological sampling. ${ }^{13}$ The main intervention was surgical removal of the fibrotic mass and insertion of a branched homograft to relieve severe stenoses of the right main pulmonary artery and SVC. ${ }^{13}$ Fijolek et al treated their patient who presented with recurrent haemoptysis, with two courses of anti-TB therapy prior to the emergence of the diagnosis of FM which developed over 10 years after the initial culture-positive TB diagnosis. ${ }^{12}$ There was severe right lower pulmonary vein and left pulmonary artery narrowing. She underwent a trial of 2 months of $60 \mathrm{mg}$ prednisolone with no surgical intervention, but the mass increased in size, and therapy was stopped..$^{12}$ However, interestingly they reported that her symptoms had not recurred at follow-up at time of publication. Zhang and colleagues removed a fibrotic mass, which encroached onto the aorta, pulmonary artery and left atrium, followed by anti-TB drugs, with good resolution of symptoms, although the length of follow-up was not specified. ${ }^{14}$ Despite compression of vascular structures, collateral circulation often develops over time and without acute obstruction, Baikoussis et al decided to monitor rather than intervene. ${ }^{10}$

In the case series reported by Mole et al, 9 out of 18 cases of FM had previous TB. ${ }^{15}$ Unfortunately, the authors do not specify outcomes specifically for the TB patients but for the whole cohort. Moreover, data for treatment with steroids or anti-TB medication was unavailable. ${ }^{15}$ Several patients underwent surgery, including superior vena cava bypass grafting, lobectomy and excision of fibrous tissue. Follow-up of 12 of the patients was for between 5 and 20 years, with 10 of these free from symptoms, including 3 that had undergone surgery for vena cava obstruction. ${ }^{15}$ The two that died had developed malignancy and did not die of complications of mediastinal fibrosis.

For those patients that have FM, not related to TB, treatment and outcomes are similar. Peikert $e t$ al reviewed 80 cases, with most of the patients having had evidence of previous Histoplasmosis infection from the Mayo Clinic between 1998 and 2007. ${ }^{19}$ A total of 31 patients had antifungal treatment, 5 received steroids and 2 trialled tamoxifen. Radiological intervention with vascular angioplasty/stenting or endobronchial balloon dilatation/ stenting was carried out in 15 patients, while 17 required surgical intervention. ${ }^{19}$ Of the 34 patients followed up from having medical therapy, less than a fifth (18\%) had some response in symptoms or radiological findings; only three patients had imaging response to antifungal therapy; and one patient had response to anti-inflammatory treatment. ${ }^{19}$ However, 21 medically treated patients remained stable. Those that underwent angioplasty or stenting required a repeat procedure within 6 to 12 months. Five out of the 12 patients who were followed up after surgery needed further intervention such as stenting or embolisation for symptom recurrence. ${ }^{19}$ However, overall survival was similar to age-matched controls, with only two patients dying as a direct result of FM. Surgery should be used as a last resort as demonstrated in one recent study, with complications from carinal pneumonectomy leading to death in 3 patients out of 18 who underwent surgical interventions relating to vital airway or lung structures. ${ }^{30}$ 
Steroid therapy has been successful in some reports. ${ }^{31}$ Sherrick et al reported that three out of six patients with diffuse radiological change responded to steroids on repeated imaging, while none of the four patients with localised fibrosis improved. ${ }^{20}$ They proposed that widespread fibrosis may be indicative of a more generalised active immunological response and therefore have a better response to anti-inflammatory therapy. ${ }^{20}$

Tamoxifen, a selective oestrogen receptor modulator, has been used in combination with steroids based on its antifibrotic effects against transforming growth factor $\beta$ activity. ${ }^{32}$ Savelli et al used steroid and tamoxifen in combination with good effect initially, and when tamoxifen was stopped disease progression recurred, before regressing once again with combination therapy. ${ }^{32}$ In contrast, when relapse occurred following weaning doses of steroids as part of combined therapy with tamoxifen in another patient, the team increased steroid doses to induce remission again with good success. ${ }^{29}$ In another case report, a unique triple combination of itraconazole, tamoxifen and high-dose steroids was successful in reducing FDG activity on PET scanning. ${ }^{28}$

\section{DISCUSSION}

Our patient had several factors contributing to his breathlessness, namely mild pulmonary hypertension, recurrent pleural effusion and parenchymal lung damage post-TB infection. The onset of mediastinal fibrosis was several years after fully treated active TB infection, and was diagnosed on CT scan, the imaging modality of choice. FDG-PET helped to establish activity within the mass, but biopsy was still required to exclude malignancy and recurrence of TB. We performed endobronchial ultrasound-guided biopsy which has the advantage of being less invasive compared with a video-assisted thorascopic surgical (VATS) biopsy or mediastinoscopy, but not all lesions are accessible via this approach. It is important to note that in our case the biopsy showed fibrous tissue without active granulomatous inflammation, and along with FDG-PET avidity, we postulate this was active fibrotic inflammation.

Treatment was initiated with high-dose steroids, and in line with other cases treated in the literature, TB chemoprophylaxis was given. ${ }^{12-14}$ Our patient had marked symptomatic relief after starting steroids, but relapse of severe dyspnoea occurred within 3 months, even though the steroid dose was only reduced from 60 to $50 \mathrm{mg}$. However, high-dose steroids with intravenous therapy again produced symptomatic relief. We did not perform a repeat FDG-PET to assess if there was a reduction in the activity within the mass, as the size of the lesion was thought to be the main factor in causing compression of local structures rather than the amount of active inflammation.

The use of MMF in our case as adjunct therapy was based on success with this therapy in treating retroperitoneal fibrosis, where Scheel et al showed that $89 \%$ of their patients had an over $25 \%$ reduction in size of the periaortic mass ${ }^{33}$ but also as a steroid-sparing agent given his diabetes. Surgical and radiological intervention was considered too high risk. The decision to operate should be reserved as a last resort, and moreover does not prevent progression of disease, with repeated procedures likely needed within $6-12$ months $^{19}$.

The relationship between TB and mediastinal fibrosis is still poorly understood, and further reporting of cases is required. In this article, we have highlighted the two forms of mediastinitis-granulomatous versus fibrous. It remains unclear whether the granulomatous form inevitably leads to the more widespread fibrous reaction. No studies exist that observe the natural course of disease, and notably this case illustrates the possibility that TB antigen may be the cause of ongoing inflammatory changes even post-treatment. The pathogenesis of TB-related FM is still speculative and partly relies on our understanding of histoplasmosis-related FM. However, it is not known if TB-related FM runs a similar clinical course to histoplasmosis. It is reasonable to trial treatments that have been successful in idiopathic or histoplasmosis-related FM in cases of post-TB mediastinal fibrotic disease.

Although our patient did not show improvement with MMF despite positive outcomes in retroperitoneal fibrosis,${ }^{33}$ we have been able to maintain clinical improvement on a combination of MMF and low-dose corticosteroid. Inoue and colleagues demonstrated the first case of IgG4-related FM with high serum IgG4 level correlating to histological findings. ${ }^{34}$ A good response to steroids was indeed observed, and the authors suggest serum IgG4 levels as an indicator for inducing steroid therapy in this specific group of patients. ${ }^{34}$ In addition, other agents such as tamoxifen or even B-lymphocyte depleting treatment may be considered on a case-to-case basis. $^{19}$

We have reviewed TB-related FM, and this should be considered in cases of FM with prior or active TB.

Contributors ZW and OMK are responsible for the conception, drafting, revision and final approval of the article. HJ, LSH and CW are responsible for reviewing the article, making critical amendments and were directly involved with the clinical care of the patient.

Funding OMK is supported by the Biomedical Research Centre.

Competing interests None declared.

Patient consent Obtained.

Provenance and peer review Not commissioned; externally peer reviewed.

Data sharing statement Due to patient confidentiality, there is limited further data, but may be available by contacting the corresponding author.

Open Access This is an Open Access article distributed in accordance with the Creative Commons Attribution Non Commercial (CC BY-NC 4.0) license, which permits others to distribute, remix, adapt, build upon this work non-commercially, and license their derivative works on different terms, provided the original work is properly cited and the use is non-commercial. See: http://creativecommons.org/ licenses/by-nc/4.0/

(c) Article author(s) (or their employer(s) unless otherwise stated in the text of the article) 2017. All rights reserved. No commercial use is permitted unless otherwise expressly granted. 


\section{REFERENCES}

1. Loyd JE, Tillman BF, Atkinson JB, et al. Mediastinal fibrosis complicating histoplasmosis. Medicine 1988;67:295-310.

2. Wheat LJ, Slama TG, Eitzen HE, et al. A large urban outbreak of histoplasmosis: clinical features. Ann Intern Med 1981:94:331-7.

3. Schowengerdt CG, Suyemoto R, Main FB. Granulomatous and fibrous mediastinitis. a review and analysis of 180 cases. $J$ Thorac Cardiovasc Surg 1969;57:365-79.

4. Chatterjee D, Bal A, Singhal M, et al. Fibrosing mediastinitis due to Aspergillus with dominant cardiac involvement: report of two autopsy cases with review of literature. Cardiovasc Pathol 2014;23:354-7.

5. Wightman SC, Kim AW, Proia LA, et al. An unusual case of Aspergillus fibrosing mediastinitis. Ann Thorac Surg 2009;88:1352-4.

6. Peikert T, Shrestha B, Aubry MC, et al. Histopathologic overlap between fibrosing mediastinitis and IgG4-related disease. Int $J$ Rheumatol 2012;2012:1-7.

7. Dehner LP, Coffin CM. Idiopathic fibrosclerotic disorders and other inflammatory pseudotumors. Semin Diagn Pathol 1998;15:161-73.

8. Fatourechi MM, Hay ID, Mclver B, et al. Invasive fibrous thyroiditis (riedel thyroiditis): the mayo clinic experience, 1976-2008. Thyroid 2011;21:765-72.

9. Parish JM, Rosenow EC. Mediastinal granuloma and mediastinal fibrosis. Semin Respir Crit Care Med 2002;23:135-44.

10. Baikoussis NG, Argiriou O, Kratimenos T, et al. Lipomatous hypertrophy of the interatrial septum and fibrosing mediastinal lymphadenopathy causing superior vena cava obstruction. Ann Card Anaesth 2015;18:609-11.

11. Ferrer Galván M, Rodríguez Portal JA, Serrano Gorarredona MP, et al. Fibrosing mediastinitis mimicking sarcoidosis. Clin Respir 2015;9:125-8.

12. Fijołek J, Wiatr $\mathrm{E}$, Błasińska-Przerwa $\mathrm{K}$, et al. Fibrosing mediastinitis as an untypical complication of tuberculosis: case report. Pol Arch Med Wewn 2009;119:752-5.

13. Ojeifo O, Gilotra NA, Kemp CD, et al. Mediastinal fibrosis of the pulmonary artery secondary to tuberculosis. Ann Thorac Surg 2015;100:e49-e50.

14. Zhang $\mathrm{C}$, Yao M, Yu Z, et al. Rare fibrosing granulomatous mediastinitis of tuberculosis with involvement of the transverse sinus. J Thorac Cardiovasc Surg 2007:133:836-7.

15. Mole TM, Glover J, Sheppard MN. Sclerosing mediastinitis: a report on 18 cases. Thorax 1995;50:280-3.

16. Ramakantan $\mathrm{R}$, Shah P. Dysphagia due to mediastinal fibrosis in advanced pulmonary tuberculosis. AJR Am J Roentgenol 1990;154:61-3.

17. Dines DE, Payne WS, Bernatz PE, et al. Mediastinal granuloma and fibrosing mediastinitis. Chest 1979;75:320-4.
18. Flieder DB, Suster S, Moran CA. Idiopathic fibroinflammatory (fibrosing/sclerosing) lesions of the mediastinum: a study of 30 cases with emphasis on morphologic heterogeneity. Mod Pathol 1999;12:257-64.

19. Peikert T, Colby TV, Midthun DE, et al. Fibrosing mediastinitis: clinical presentation, therapeutic outcomes, and adaptive immune response. Medicine 2011;90:412-23.

20. Sherrick AD, Brown LR, Harms GF, et al. The radiographic findings of fibrosing mediastinitis. Chest 1994;106:484-9.

21. McNeeley MF, Chung JH, Bhalla S, et al. Imaging of granulomatous fibrosing mediastinitis. AJR Am J Roentgenol 2012;199:319-27.

22. Atasoy C, Fitoz S, Erguvan B, et al. Tuberculous fibrosing mediastinitis: CT and MRI findings. J Thorac Imaging 2001;16:191-3.

23. Rholl KS, Levitt RG, Glazer HS. Magnetic resonance imaging of fibrosing mediastinitis. AJR Am J Roentgenol 1985;145:255-9.

24. Mallin WH, Silberstein EB, Shipley RT, et al. Fibrosing mediastinitis causing nonvisualization of one lung on pulmonary scintigraphy. Clin Nucl Med 1993;18:594-6.

25. Savolaine ER, Schlembach PJ. Scintigraphy compared to other imaging modalities in benign superior vena caval obstruction accompanying fibrosing mediastinitis. Clin Imaging 1989;13:234-8.

26. Park HM, Jay SJ, Brandt MJ, et al. Pulmonary scintigraphy in fibrosing mediastinitis due to histoplasmosis. J NuCl Med 1981;22:349-51.

27. Chong S, Kim TS, Kim BT, et al. Fibrosing mediastinitis mimicking malignancy at CT: negative FDG uptake in integrated FDG PET/CT imaging. Eur Radiol 2007:17:1644-6.

28. Takalkar AM, Bruno GL, Makanjoula AJ, et al. A potential role for F-18 FDG PET/CT in evaluation and management of fibrosing mediastinitis. Clin Nucl Med 2007;32:703-6.

29. Kaya H, Rider K, Cho AH, et al. The role of PET scan in monitoring the progression of fibrosing mediastinitis. Clin Imaging 2016;40:177-9.

30. Mathisen DJ, Grillo HC. Clinical manifestation of mediastinal fibrosis and histoplasmosis. Ann Thorac Surg 1992;54:1053-8.

31. Ikeda K, Nomori H, Mori T, et al. Successful steroid treatment for fibrosing mediastinitis and sclerosing cervicitis. Ann Thorac Surg 2007;83:1199-201.

32. Savelli BA, Parshley M, Morganroth ML. Successful treatment of sclerosing cervicitis and fibrosing mediastinitis with tamoxifen. Chest 1997:111:1137-40.

33. Scheel PJ, Sozio SM, Feeley N. Medical management of retroperitoneal fibrosis. Trans Am Clin Climatol Assoc 2012;123:283-90.

34. Inoue M, Nose $\mathrm{N}$, Nishikawa $\mathrm{H}$, et al. Successful treatment of sclerosing mediastinitis with a high serum IgG4 level. Gen Thorac Cardiovasc Surg 2007;55:431-3. 